\title{
Various Magnetic Resonance Imaging Patterns of Chronic Subdural Hematomas: Indicators of the Pathogenesis?
}

\author{
Hirosuke FujISAWA, Sadahiro NOMURA, Koji KAJIWARA, Shoichi KATO, \\ Masami FUJII, and Michiyasu SUZUKI \\ Department of Neurosurgery, Clinical Neuroscience, Yamaguchi University \\ School of Medicine, Ube, Yamaguchi
}

\begin{abstract}
Magnetic resonance (MR) imaging can detect various patterns in chronic subdural hematomas. These patterns were compared to the computed tomography (CT) appearances and chemical analysis of the content in 60 hematomas from 44 patients. The hematomas could be classified into five types on both $T_{1^{-}}$and $T_{2^{-}}$-weighted images: low, high, and mixed intensity, isointensity, and layered. Combining the $T_{1^{-}}$and $T_{2}$-weighted images of all 60 hematomas revealed a total of 14 different imaging patterns. Combining the CT and MR imaging findings of 55 hematomas identified 25 different patterns. Analysis of the hematoma contents showed that hemolysis-related parameters, such as potassium, glutamate oxaloacetate transaminase, bilirubin, lactate dehydrogenase, and protein concentration, were markedly higher than in the peripheral blood, and there were significant correlations between these parameters. Mixed intensity hematomas were significantly thicker than the other types, and showed markedly higher values of hemolysis-related parameters. Factors affecting the CT and MR imaging findings, such as fresh bleeding, hemolysis, and hemoglobin changes, coexist in a hematoma to varying degrees, and these factors may interact with the age of the hematoma to produce the different patterns that are observed.
\end{abstract}

Key words: chronic subdural hematoma, magnetic resonance imaging, computed tomography, hemolysis, pathogenesis

\section{Introduction}

The pathogenesis of chronic subdural hematoma (CSH) is still not fully understood despite many histological, chemical, and physiological investigations. CSH may manifest as various clinical symptoms, such as headache, hemiparesis, and sometimes consciousness disturbance. The diagnosis is usually based only on computed tomography (CT), and surgery is performed in the early stage. Since the information provided by CT is usually sufficient for surgery, preoperative magnetic resonance (MR) imaging is not carried out in most patients with $\mathrm{CSH}$. However, MR imaging is an important diagnostic tool for CSH, especially cases that appear on CT as bilateral isodense and multilayered hematomas, and for the differential diagnosis between hematoma and hygroma or empyema. ${ }^{5}$ MR imaging can also provide important information concerning the nature of the hematoma. We have

Received May 26, 2005; Accepted February 3, 2006 noticed that CSHs have various characteristics on MR imaging, and that there is no constant relationship between the CT and MR imaging findings. The MR imaging features of $\mathrm{CSH}$ have been described, ${ }^{5,16,19)}$ but the relationship of the MR imaging findings with the pathogenesis has not been considered.

The present study examined the various MR imaging patterns of CSHs and their relationship to the CT findings, as well as the chemical analysis of the hematoma contents.

\section{Materials and Methods}

Forty-four patients with $\mathrm{CSH}, 35$ males and nine females aged 5-97 years (mean 69.8 years), were studied. None had received previous treatment for $\mathrm{CSH}$. Twenty-eight patients had unilateral hematomas and 16 had bilateral hematomas. The total number of hematomas examined was 60. Preoperative CT was performed in all patients, and MR imaging was also performed as soon as possible 

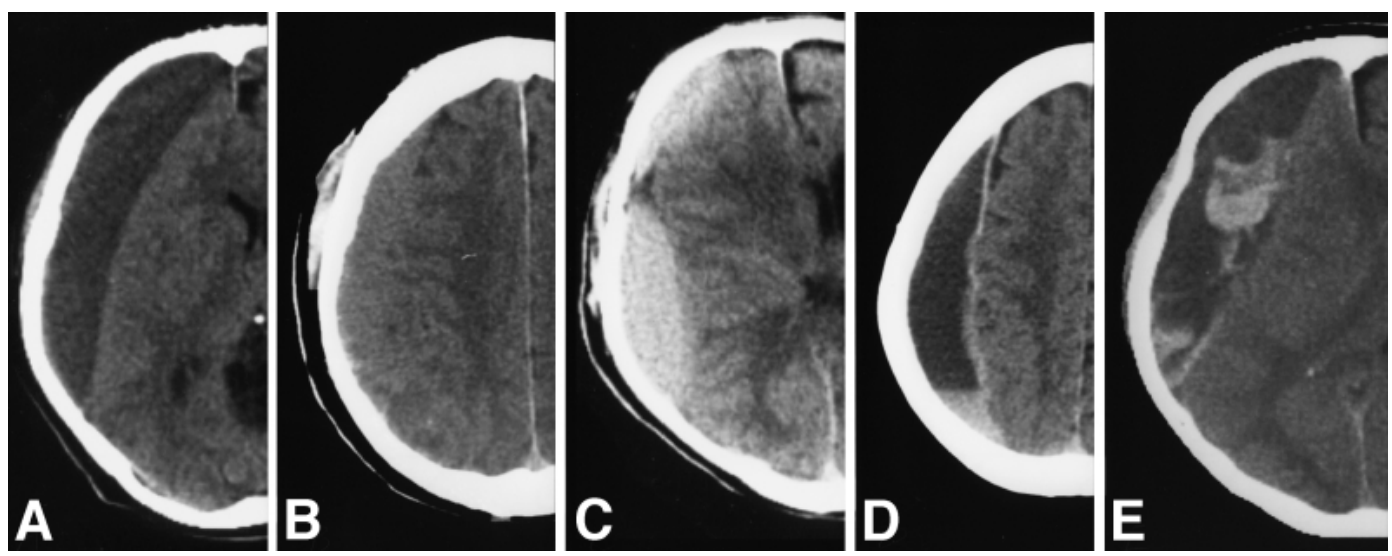

Fig. 1 Computed tomography appearance of chronic subdural hematoma. A: low density, B: isodensity, C: high density, D: layered, E: mixed density.
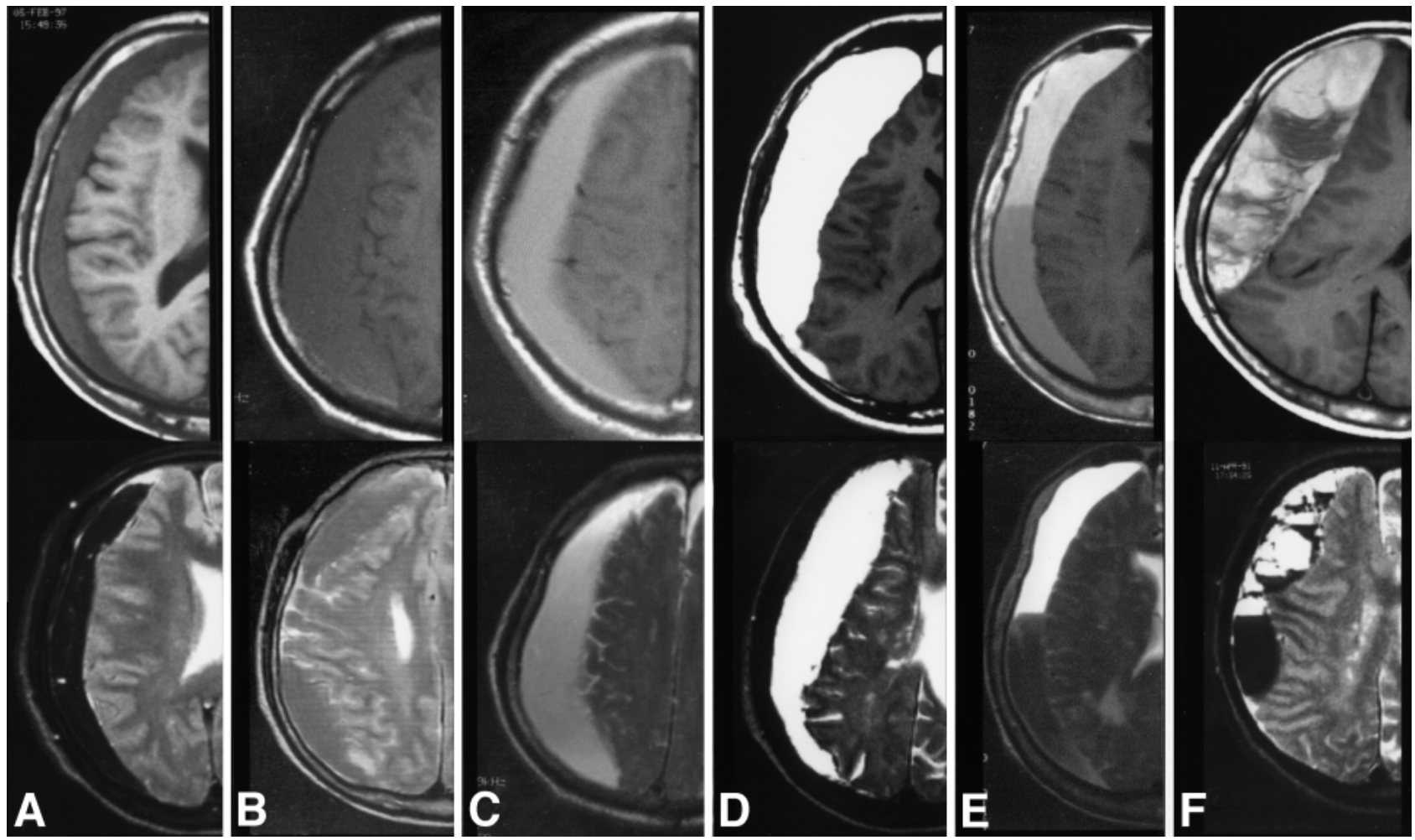

Fig. 2 Magnetic resonance imaging appearance of chronic subdural hematoma. The hematomas were classified into five types: low intensity (A), isointensity (B), high intensity (C, D), layered (E), and mixed intensity (F) on both $T_{1}$ - (upper row) and $T_{2}$-weighted (lower row) images. The high intensity type could be subdivided into the moderately high (C) and very high intensity (D) types.

thereafter. CT and MR imaging were performed within 9 days in 40 patients with 55 hematomas.

The MR imaging patterns of all 60 hematomas were first analyzed. However, MR imaging findings may change with time, so too long a delay might mean that the MR imaging and CT findings are no longer comparable. Therefore, the two patients for whom there was a time lag of 16 and 18 days were excluded, and the relationships between CT and MR imaging findings of 55 hematomas were analyzed. 
The hematoma contents and samples of peripheral blood from 21 patients with 26 hematomas were analyzed. Peripheral blood samples were taken from the patients on admission. The CSHs were aspirated by puncturing the dura mater with a disposable plastic syringe. All hematomas were typical, liquefied dark-red hematomas with subdural membranes. White and red blood cell (WBC and RBC) counts and hemoglobin levels in both hematomas and peripheral blood were measured. The following parameters were measured routinely from the peripheral blood on admission, and in the hematoma samples: protein, albumin, globulin, blood sugar, cholesterol, blood urea nitrogen, creatinine, uric acid, choline esterase, glutamate oxaloacetate transaminase (GOT), glutamate pyruvate transaminase, alkaline phosphatase, $\gamma$-glutamyl transpeptidase, direct and indirect bilirubin, and lactate dehydrogenase (LDH).

Data are expressed as the mean \pm standard deviation. The statistical significance between two groups was assessed using the unpaired Student's $t$ test. Correlations between different parameters were examined using Pearson's linear regression. Differences were considered to be statistically significant at $\mathrm{p}<0.05$.

\section{Results}

The CT appearance of CSHs could be classified into five types: low, high, and mixed density, isodensity, and layered (Fig. 1). The $\mathrm{T}_{1^{-}}$and $\mathrm{T}_{2}$-weighted $\mathrm{MR}$ imaging appearances could also be classified into five types: low, high, and mixed intensity, isointensity (where the intensity of the hematoma appears equal to that of the brain), and layered (Fig. 2). Although the high intensity type could be further subdivided into moderately high and very high intensity types, these two subtypes were considered together in this study.

The $\mathrm{T}_{1}$ - and $\mathrm{T}_{2}$-weighted MR images of 60 hematomas revealed a total of 14 different patterns, and the combination of CT scans and MR images of 55 hematomas revealed 25 different patterns (Table 1). Interestingly, there were some hematomas for which CT exhibited a homogeneous pattern (low density or isodensity), whereas MR imaging exhibited a heterogeneous pattern (mixed intensity or layered). CT showed layered CSH in one case, whereas $\mathrm{T}_{1}$ - and $\mathrm{T}_{2}$-weighted images showed isointensity and high intensity $\mathrm{CSH}$, respectively. Hematomas appearing as mixed density on CT always appeared as mixed intensity on either $T_{1^{-}}$or $T_{2}$-weighted images, or both.

Levels of potassium (hematomas $5.0 \pm 1.7 \mathrm{mmol} / \mathrm{l}$,
Table 1 Patterns on computed tomography (CT) and magnetic resonance imaging

\begin{tabular}{|c|c|c|c|c|}
\hline Pattern & $\mathrm{CT}$ & $\mathrm{T} 1$ & $\mathrm{~T} 2$ & $\begin{array}{c}\text { No. of } \\
\text { hematomas }\end{array}$ \\
\hline 1 & low & low & high & 4 \\
\hline 2 & low & high & high & 3 \\
\hline 3 & low & high & layered & 1 \\
\hline 4 & low & high & low & 1 \\
\hline 5 & low & high & mixed & 1 \\
\hline 6 & low & iso & iso & 1 \\
\hline 7 & low & mixed & mixed & 1 \\
\hline 8 & iso & layered & layered & 2 \\
\hline 9 & iso & mixed & mixed & 2 \\
\hline 10 & iso & iso & low & 1 \\
\hline 11 & iso & high & low & 1 \\
\hline 12 & iso & high & iso & 1 \\
\hline 13 & high & high & high & 4 \\
\hline 14 & high & high & low & 2 \\
\hline 15 & high & high & iso & 2 \\
\hline 16 & high & iso & high & 1 \\
\hline 17 & high & low & high & 1 \\
\hline 18 & layered & layered & layered & 6 \\
\hline 19 & layered & high & layered & 1 \\
\hline 20 & layered & low & layered & 1 \\
\hline 21 & layered & iso & layered & 1 \\
\hline 22 & layered & iso & high & 1 \\
\hline 23 & mixed & mixed & mixed & 12 \\
\hline 24 & mixed & high & mixed & 3 \\
\hline 25 & mixed & mixed & low & 1 \\
\hline Total & & & & 55 \\
\hline
\end{tabular}

peripheral blood $4.1 \pm 0.4 \mathrm{mmol} / \mathrm{l}$,), GOT (hematomas $92.9 \pm 75.8 \mathrm{IU} / \mathrm{l}$, peripheral blood $38.0 \pm 42.8$ IU/l), bilirubin (hematomas $10.9 \pm 5.3 \mathrm{mg} / \mathrm{dl}$, peripheral blood $0.8 \pm 0.4 \mathrm{mg} / \mathrm{dl}$ ), and LDH (hematomas $1649 \pm 1489 \mathrm{IU} / \mathrm{l}$, peripheral blood $211 \pm 85$ IU/l) were all significantly higher in the hematomas than in the peripheral blood. Protein concentrations were also higher in the hematomas (hematomas 7.2 $\pm 2.5 \mathrm{~g} / \mathrm{dl}$, peripheral blood $6.4 \pm 0.6 \mathrm{~g} / \mathrm{dl}$ ), although the difference was not statistically significant. All these parameters showing markedly higher values in the hematoma were hemolysis-related. There were significant correlations between LDH and indirect bilirubin $(\mathrm{r}=0.53, \mathrm{p}<0.05)$, GOT $(\mathrm{r}=$ $0.61, \mathrm{p}<0.005)$, and total protein $(\mathrm{r}=0.86, \mathrm{p}<$ 0.0001). There were also significant correlations between the amount of total protein and indirect bilirubin $(\mathrm{r}=0.56, \mathrm{p}<0.01)$.

Table 2 shows the differences between the mixed intensity hematomas on $\mathrm{T}_{1^{-}}$or $\mathrm{T}_{2}$-weighted $\mathrm{MR}$ images and the other types of hematoma (low intensity, isointensity, high intensity, and layered). The mixed intensity hematomas were significantly 
Table 2 Comparison between hematomas of mixed intensity and other types

\begin{tabular}{|c|c|c|c|c|}
\hline & Magnetic resonance imaging type & $\mathrm{n}$ & Values & $\mathrm{p}$ Value \\
\hline Hematoma thickness (mm) & others & 19 & $19.5 \pm 6.1$ & \\
\hline Total protein (g/dl) & others & 15 & $6.3 \pm 1.9$ & \\
\hline \multirow[t]{2}{*}{ Indirect bilirubin $(\mathrm{mg} / \mathrm{dl})$} & mixed intensity & 8 & $11.4 \pm 4.6$ & $0.0212^{*}$ \\
\hline & others & 12 & $6.6 \pm 3.8$ & \\
\hline Lactate dehydrogenase (IU/l) & others & 15 & $1076 \pm 899$ & \\
\hline \multirow[t]{2}{*}{ Red blood cell $\left(\times 10^{4} / \mathrm{l}\right)$} & mixed intensity & 10 & $279 \pm 189$ & 0.3671 \\
\hline & others & 16 & $347 \pm 181$ & \\
\hline \multirow[t]{2}{*}{ Hemoglobin (g/dl) } & mixed intensity & 7 & $10.9 \pm 4.3$ & 0.7684 \\
\hline & others & 12 & $11.7 \pm 5.4$ & \\
\hline White blood cell $(/ \mathrm{l})$ & mixed intensity & 9 & $5722 \pm 3703$ & 0.6820 \\
\hline
\end{tabular}

Values are the mean \pm standard deviation. Significant difference (Student's $t$ test): ${ }^{*} \mathrm{p}<0.05, \uparrow \mathrm{p}<0.005$.

thicker than the other types $(p=0.0038)$. The total protein and indirect bilirubin levels were significantly higher in the mixed intensity hematomas than in the other types $(\mathrm{p}=0.0385$ and 0.0212 , respectively). The $\mathrm{LDH}$ levels were also apparently higher in mixed intensity hematomas than in the other types, although not significantly ( $p=0.0518$ ). No significant differences were seen in the levels of RBCs, WBCs, and hemoglobin, which are regarded as indices of hemorrhage from the membrane into the hematoma cavity. There were also no differences in the concentration of albumin between hematoma types, which is regarded as an index of serum exudation from the membrane.

\section{Discussion}

This study demonstrates that the MR imaging appearance of CSHs includes a variety of patterns. Since the CT, and $\mathrm{T}_{1}$-weighted and $\mathrm{T}_{2}$-weighted images could all be classified into five types, the theoretical number of combinations of CT and MR imaging patterns is $125(5 \times 5 \times 5)$. In this study, the combinations of appearances of 55 hematomas were classified into 25 patterns. If more hematomas had been examined, it is possible that the number of patterns would have increased.

The hematoma cavity is encapsulated by the inner and outer subdural membranes. The outer membranes consist of granulation tissue, which is a type of reparative or inflammatory tissue including collagen fibrils, fibroblasts, and abundant capillaries (macrocapillaries or sinusoids). ${ }^{1)}$ Endothelial fenes- trations and open gaps between adjacent endothelial cells of the macrocapillaries have also been observed. ${ }^{20)}$ The hematoma is typically dark red and liquefied, and fresh bleeding into the hematoma cavity can occur. ${ }^{10)}$ The hematoma includes high concentrations of fibrinolytic parameters such as tissue-type plasminogen activator, ${ }^{7)}$ plasmin- $\alpha_{2}$ plasmin inhibitor complex, ${ }^{15)}$ and fibrin and fibrinogen degradation products. ${ }^{9,11)}$ On the other hand, clotting parameters are markedly reduced in hematomas. ${ }^{1,11)}$ Various theories have been proposed to explain the enlargement of CSHs, but intermittent cycles of bleeding, coagulation, fibrinolysis, and rebleeding from the highly vascularized outer membrane are widely accepted to be responsible. . $^{2,711,15,18)}$ Increased vascular permeability and subsequent serum exudation from the outer membrane may also be involved in hematoma progression, supported by the observation that radioisotope-labeled albumin enters the hematoma cavity. ${ }^{1,3,20)}$

Subdural hygroma following minor head injury sometimes develops into $\mathrm{CSH},{ }^{13,14)}$ and this process can be visualized as a gradual increase in hematoma density on CT. However, bleeding into the hematoma cavity may not be the only factor in such changes. ${ }^{3)}$ In the present study, chemical analysis of the hematoma contents demonstrated markedly high values and significant correlations between hemolysis-related parameters (i.e. potassium, GOT, bilirubin, LDH, and protein). After bleeding into the hematoma cavity, RBCs and hemoglobin persist for a considerable period until symptoms develop. Hemolysis then proceeds within the hematoma, 
which causes an increase in protein concentration and may affect the CT density or $\mathrm{T}_{1}$ - and $\mathrm{T}_{2}$-weighted intensity. Within the hematoma, hemoglobin gradually changes into oxyhemoglobin, deoxyhemoglobin, and methemoglobin. In fact, hemoglobin breakdown products from the hematoma migrate with the alpha 2- and beta-globulins, ${ }^{17)}$ and the protein fraction in CSH shows high alpha 2- and betaglobulin ratios. ${ }^{3)}$ The severity of these changes may differ between hematomas, depending on the hematoma age. Therefore, many factors affecting CT and MR imaging findings, such as fresh bleeding, hemolysis, and hemoglobin changes, coexist in the hematoma to varying degrees. These factors may interact with the age of the hematoma, a parameter that appears to be most discernible in the mixed intensity hematomas. Therefore, we investigated whether the contents of the mixed intensity hematomas had similar characteristics.

The mixed intensity hematomas were typically dark red and liquefied, as confirmed during surgery. There were no differences between the mixed intensity hematomas and other types of hematoma with respect to the amounts of RBCs and hemoglobin, which are indices of bleeding, and the albumin concentration, which is an index of serum exudation. In contrast, the hemolysis-related parameters (protein, indirect bilirubin, LDH) were higher in the mixed intensity hematomas. In general, hematoma thickness increased with hematoma age, and mixed intensity hematomas were significantly thicker than the other types. In the present study, the findings of CT scans, and $\mathrm{T}_{1}$-weighted and $\mathrm{T}_{2}$-weighted images were dissociated in some CSHs. These differences may be attributable to the above-mentioned complicating factors coexisting within the hematoma. Although a phantom study using CT or MR imaging seems to be an attractive method for clarifying sequential changes in $\mathrm{CSH}$, it may actually be difficult to reproduce all of the above-mentioned phenomena proceeding at the same time within the hematoma.

Hemolysis and hemoglobin changes may lead to an increase in colloidal osmotic pressure within a hematoma. An early hypothesis in 1932 suggested that an increase in the osmolality of the hematoma contents causes enlargement of $\mathrm{CSH}$, and some other investigators supported this idea. ${ }^{4,6,12,21)}$ Some years later, this hypothesis was refuted by demonstrating that no differences in osmolality or colloidal osmotic pressure between the hematoma contents and the peripheral blood. ${ }^{17)}$ In contrast, using a different measurement method, hematomas were found to have a high colloidal osmotic pressure that gradually increased with time, and this increase might be at least partly responsible for $\mathrm{CSH}$ enlargement. ${ }^{8)}$ However, the differences in hematoma types evident on CT and MR imaging were not considered. The results of the present study may draw renewed attention to the osmotic pressure theory.

\section{References}

1) Fujisawa $H$, Ito $H$, Kashiwagi $S$, Nomura $S$, Toyosawa $\mathrm{M}$ : Kallikrein-kinin system in chronic subdural haematomas: its roles in vascular permeability and regulation of fibrinolysis and coagulation. J Neurol Neurosurg Psychiatry 59: 388-394, 1995

2) Fujisawa $H$, Ito $H$, Saito $K$, Ikeda $K$, Nitta $H$, Yamashita J: Immunohistochemical localization of tissue-type plasminogen activator in the lining wall of chronic subdural hematoma. Surg Neurol 35: 441-445, 1991

3) Fujisawa H, Nomura S, Tsuchida E, Ito H: Serum protein exudation in chronic subdural haematomas: a mechanism for haematoma enlargement? Acta Neurochir (Wien) 140: 161-166, 1998

4) Gardner WJ: Traumatic subdural hematoma with particular reference to the latent interval. Arch Neurol Psychiat 27: 847-858, 1932

5) Guenot M: [Chronic subdural hematoma: diagnostic imaging studies]. Neurochirurgie 47: 473-478, 2001 (Fre, with Eng abstract)

6) Ingraham FD, Matson DD: Subdural hematoma in infancy. J Pediat 24: 1-37, 1944

7) Ito H, Saito K, Yamamoto S, Hasegawa T: Tissue-type plasminogen activator in the chronic subdural hematoma. Surg Neurol 30: 175-179, 1988

8) Ito H, Shimoji T, Yamamoto S, Saito K, Uehara S: Colloidal osmotic pressure in chronic subdural hematoma. Neurol Med Chir (Tokyo) 28: 650-653, 1988

9) Ito H, Yamamoto S, Komai T, Mizukoshi H: Role of hyperfibrinolysis in the etiology of chronic subdural haematoma. J Neurosurg 45: 26-31, 1976

10) Ito $H$, Yamamoto $S$, Saito $K$, Ikeda K, Hisada K: Quantitative estimation of hemorrhage in chronic subdural haematoma using the ${ }^{51} \mathrm{Cr}$ erythrocyte labeling method. J Neurosurg 66: 862-864, 1987

11) Kawakami Y, Chikama M, Tamiya T, Shimamura $Y$ : Coagulation and fibrinolysis in chronic subdural hematoma. Neurosurgery 25: 25-29, 1989

12) Munro D, Merritt HH: Surgical pathology of subdural hematoma. Based on a study of one hundred and five cases. Arch Neurol Psychiat 35: 64-78, 1936

13) Ohno K, Suzuki R, Masaoka H, Matsushima Y, Inaba Y, Monma S: Chronic subdural haematoma preceded by persistent traumatic subdural fluid collection. J Neurol Neurosurg Psychiatry 50: 1694-1697, 1987

14) Park CK, Choi KH, Kim MC, Kang JK, Choi CR: Spontaneous evolution of posttraumatic subdural hygroma into chronic subdural haematoma. Acta Neurochir (Wien) 127: 41-47, 1994 
15) Saito K, Ito $H$, Hasegawa T, Yamamoto S: Plasmin- $\alpha_{2}$ plasmin inhibitor complex and $\alpha_{2}$-plasmin inhibitor in chronic subdural hematoma. J Neurosurg 70: 68-72, 1989

16) Tsutsumi K, Maeda K, Iijima A, Usui M, Okada Y, Kirino T: The relationship of preoperative magnetic resonance imaging findings and closed system drainage in the recurrence of chronic subdural hematoma. J Neurosurg 87: 870-875, 1997

17) Weir B: The osmolality of subdural hematoma fluid. J Neurosurg 34: 528-533, 1971

18) Weir B, Gordon P: Factors affecting coagulation: Fibrinolysis in chronic subdural fluid collections. J Neurosurg 58: 242-246, 1983

19) Williams VL, Hogg JP: Magnetic resonance imaging of chronic subdural hematoma. Neurosurg Clin N Am 11: 491-498, 2000

20) Yamashima T, Yamamoto S, Friede RL: The role of endothelial gap junction in the enlargement of chronic subdural hematomas. J Neurosurg 59: 298-303, 1983

21) Zollinger R, Gross RE: Traumatic subdural hematomas. An explanation of late onset of pressure symptoms. JAMA 103: 245-249, 1934

Address reprint requests to: H. Fujisawa, M.D., Department of Neurosurgery, Clinical Neuroscience, Yamaguchi University School of Medicine, 1-1-1 Minamikogushi, Ube, Yamaguchi 755-8505, Japan. e-mail: hirofuji@yamaguchi-u.ac.jp

\section{Commentary}

The present study examines the relationship between MR imaging patterns and CT findings in correlation with chemical analysis of the contents of 55 chronic subdural hematomas (CSHs) in 45 patients. Combining the different CT and MR imaging findings (low-high-mixed-layered density/intensity), 25 imaging patterns were described. The majority of these patterns (15/25) were found in only one of each case; the mixed and layered signal patterns were found most frequently (in 12 and 6 cases, respectively). Chemical analysis of the hematoma contents yielded high values of hemolysis-related parameters $\left(\mathrm{K}^{+}\right.$, GOT, indirect bilirubin, $\mathrm{LDH}$ and protein) predominantly in mixed signal pattern hematomas as opposed to others. The differences reached statistical significance only for protein and bilirubin.

The authors concluded from their results support for the osmotic pressure theory in the pathogenesis of $\mathrm{CSH}$. However, the osmotic pressure of the hematoma content was not directly assessed in the present study, nor were there serial CT or MR imaging showing possible changes of the hematoma extension or signal pattern over time. A prospective study addressing these questions probably would shed more light on the pathogenesis of $\mathrm{CSH}$.

Gerrit FISCHER, M.D. and Axel PERnECZKY, M.D. Department of Neurosurgery Medical School Johannes Gutenberg University Mainz, Germany

Chronic subdural hematoma (CSDH) is one of the most common clinical entities in daily neurosurgical practice. In the past two decades, magnetic resonance (MR) imaging pattern of CSDH has been reported. CSDHs are hyperintense on both T1- and T2-weighted MR images, in general. Some are iso- or hypointense on T1-weighted images. ${ }^{11}$ Iso- or hypointense on T1and hypointense on T2-weighted images are short in terms of appearance of symptoms. ${ }^{5}$ Symptomatic CSDH tends to have a black band on the inner membrane of a CSDH on T2*-weighted images. ${ }^{3)}$ Hypointense on T1- and hypointense on T2-weighted images suggests hemoglobin or methemoglobin. ${ }^{2}$ The subdural hyperintense bands on diffusion-weighted imaging are considered to be intracellular and/or extracellular methemoglobin based on T1- and T2weighted imaging and intraoperative findings. The subdural hyperintense band is an important finding indicating relatively fresh bleeding from the outer membrane. ${ }^{4)}$

Fujisawa and colleagues report on various MR imaging patterns of CSDH. Combined T1- and T2weighted imaging findings of 60 hematomas were classified into 12 patterns. Mixed intensity hematoma on T1- or T2-weighted images was significantly thicker than other patterns. The hematoma contents and peripheral blood were also analyzed. Hemolysis-related parameters, such as potassium, transaminase, bilirubin, lactate dehydrogenase, and protein concentration, were markedly higher than peripheral blood. These findings indicate hemorrhage from the membrane into the hematoma cavity. Hemolysis and hemoglobin changes may increase colloidal osmotic pressure within a hematoma. The increased osmotic pressure enlarges the volume of CSDH. Although this osmotic pressure theory was rejected in $1971,{ }^{6)}$ Fujisawa et al. suggest the bleeding, hemolysis and osmotic change theory again.

\section{References}

1) Hosoda K, Tamaki N, Masumura M, Matsumoto $S$, Maeda F: Magnetic resonance images of chronic subdural hematomas. J Neurosurg 67: 677-683, 1987

2) Imaizumi T, Horita Y, Honma T, Niwa J: Association 
between a black band on the inner membrane of a chronic subdural hematoma on $\mathrm{T} 2{ }^{*}$-weighted magnetic resonance images and enlargement of the hematoma. J Neurosurg 99: 824-830, 2003

3) Kaminogo M, Moroki J, Ochi A, Ichikura A, Onizuka M, Shibayama A, Miyake H, Shibata S: Characteristics of symptomatic chronic subdural haematomas on highfield MRI. Neuroradiology 41: 109-116, 1999

4) Kuwahara S, Fukuoka M, Koan Y, Miyake H, Ono Y, Moriki A, Mori K, Mokudai T, Uchida Y: Subdural hyperintense band on diffusion-weighted imaging of chronic subdural hematoma indicates bleeding from the outer membrane. Neurol Med Chir (Tokyo) 45: 125-131, 2005

5) Tsutsumi K, Maeda K, Iijima A, Usui M, Okada Y, Kirino $T$ : The relationship of preoperative magnetic resonance imaging findings and closed system drainage in the recurrence of chronic subdural hematoma. J Neurosurg 87: 870-875, 1997

6) Weir B: The osmolality of subdural hematoma fluid. J Neurosurg 34: 528-533, 1971

Takashi HaYASHI, M.D. Department of Neurosurgery

St. Mary's Hospital Kurume, Fukuoka, Japan
Chronic subdural hematoma is one of the common neurosurgical diseases. Much research has been carried out for many years in order to understand its pathogenesis. The author has done much research and conscientious analysis. The 60 hematomas in 40 cases were classified and analyzed to produce different patterns that were observed with computed tomography (CT) appearances and chemical analysis of the content. Many factors affecting the CT and MR imaging findings, such as fresh bleeding, hemolysis and hemoglobin changes, coexist in a hematoma to varying degrees. These data suggest the complication of chronic subdural hematoma formation, and suggest we should pay attention to the osmotic pressure theory once more.

Yazhuo ZHANG, M.D. Department of Neurosurgery Beijing Neurosurgical Institute Beijing, P.R.C. 